\title{
Comparison of digital surface models for snow depth mapping with UAV and aerial cameras
}

\author{
R. Boesch ${ }^{\mathrm{a}}$, Y. Bühler ${ }^{\mathrm{b}}$, M. Marty ${ }^{\mathrm{a}}$, C.Ginzler ${ }^{\mathrm{a}}$ \\ ${ }^{a}$ Swiss Federal Institute for Forest, Snow and Landscape Research WSL, Birmensdorf, Switzerland- (ruedi.boesch, mauro.marty, \\ christian.ginzler)@wsl.ch \\ b WSL Institute for Snow and Avalanche Research SLF, Davos, Switzerland - buehler@slf.ch
}

\section{Commission VIII, WG 6}

KEY WORDS: snow height, dense point cloud, digital surface model, aerial camera

\begin{abstract}
:
Photogrammetric workflows for aerial images have improved over the last years in a typically black-box fashion. Most parameters for building dense point cloud are either excessive or not explained and often the progress between software releases is poorly documented. On the other hand, development of better camera sensors and positional accuracy of image acquisition is significant by comparing product specifications. This study shows, that hardware evolutions over the last years have a much stronger impact on height measurements than photogrammetric software releases.

Snow height measurements with airborne sensors like the ADS100 and UAV-based DSLR cameras can achieve accuracies close to GSD * 2 in comparison with ground-based GNSS reference measurements. Using a custom notch filter on the UAV camera sensor during image acquisition does not yield better height accuracies. UAV based digital surface models are very robust. Different workflow parameter variations for ADS100 and UAV camera workflows seem to have only random effects.
\end{abstract}

1.

\section{INTRODUCTION}

Deriving information on snow depth and its spatial distribution is essential for several applications in hydrology and foremost in snow and avalanche research. Using operationally available aerial images acquired with the Leica ADS80 in 2012, it could be demonstrated that snow depth measurements with $0.30 \mathrm{~m}$ RMSE can be achieved in high-alpine catchment areas (Bühler 2015a). Due to the high radiometric resolution of the images (12 bits) and the use of the near infrared band (NIR), images were not saturated over bright, snow-covered areas and required texture could be identified even in shadow areas. The NIR band was also used for snow type mapping catchments (Bühler et al. 2015b). Using 2 different photogrammetric software suites for ADS80-data, SOCET SET ATE (automatic terrain extraction) and SOCET SET NGATE (next geneneration automatic terrain extraction), accuracy results were partially comparable.

In recent years Leica has renewed its product line with the ADS100 camera system and sensor head SH 100 and offers significant enhancements such as an improved radiometric resolution from 12 to 14 bits, an increased spatial resolution from $25 \mathrm{~cm}$ to $15 \mathrm{~cm}$ at the same flight height and a triple stereo capability for all spectral bands. A major restriction for the frequent use of professional aerial sensor systems to map snow height is the fact, that they are mostly operated by national or commercial agencies, though orders are expensive and require long term planning, which is often not feasible due to changing weather and snow conditions in winter time.

In contrast many different types of unmanned aerial vehicles (UAV) with a large variety of camera sensors are meanwhile available, allowing for flexible and cost effective calculation of digital surface models (DSM).

The user controlled definition of acquisition time and region of interest with UAV's is a clear advantage and allows to acquire images at optimized snow illumination and weather conditions. But the limited target area represents still a major constraint overall. Increasing the flight time for UAV's due to improved energy management and optimized flight planning can still by far not keep up with image acquisition campaigns with airplanes. But the versatile planning with a UAV allows to acquire image data for specific areas of interest and results can be combined with ADS100 results for whole catchment areas. In the last years, sensor hardware and software workflows to process and analyze digital aerial data have improved by many different vendors at irregular intervals (DeVenecia 2007, Lee 2008, Nolan 2015, Whitehead 2013, Zhang 2007).

Mainly the increased spatial resolution of the ADS100 sensor combined with improved data processing raises the question, what improvement concerning the resulting accuracy of DSM can be expected from an updated workflow for aerial images. with ADS100 data. Processing workflows for UAV images have been developed even faster over the last years, mainly due to optimized bundle adjustment during orientation and multi-view dense matching, but platform stability and reduced motion blur due to better navigation control are also important for an expected better accuracy.

This study focuses therefore on the hypothesis, that combined evolutions over the last years in hardware and software for UAV and aerial sensors are significant and therefore should be reflected in improved results.

\section{AERIAL IMAGES AND REFERENCE DATA}

To compare the performance and related accuracies for the calculation of digital surface models a field campaign has been organized in the late winter period 2015. A simultaneous flight on 15. April 2015 with UAV and airplane was realized to minimize the influence of varying illumination and weather conditions. Both flight took place between 9 and 11 am with a slightly overcast sky, which represents a nearly ideal situation for sensors comparisons. Melted "spring" snow was dominating over the whole test site. 


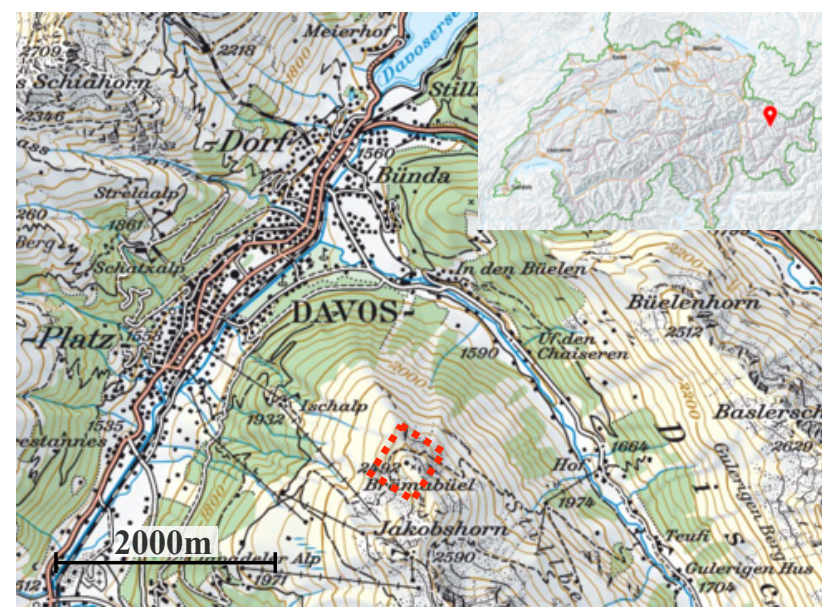

Figure 1. Test site "Brämabühl"

\subsection{Test site Brämabühl}

The test site Brämabühl is located at the top of the ski area Jakobshorn in Davos, Switzerland at an elevation of $2500 \mathrm{~m}$ a.s.l. (Figure 1). At this test site we expect a significant variability of snow depth due to high wind exposure around the top of a crest with variable slopes ranging from SW to NE. Additionally, the ski runs present within the area are typical areas for snow grooming and artificial snow production. The top of Brämabühl is covered mainly by high alpine meadow and small bushes, but no trees or larger bushes grow at this local climate.

\subsection{Acquisition flights}

The image acquisition of ADS100 data was flown within 90 minutes at an elevation of approximately $4000 \mathrm{~m}$ a.s. 1 . (1500m above the test site). The mean ground sampling distance (GSD) of the imagery varies between 0.15 and $0.157 \mathrm{~m}$, caused by the high-alpine terrain. To optimize contrast and low reflection on snow-covered areas (Bühler 2015b), the NIR bands at nadir $\left(0^{\circ}\right)$ and backward $\left(17^{\circ}\right)$ from the available $\operatorname{ADS} 100$ bands have been selected for processing.

The photogrammetric UAV missions have been performed with an Ascending Technologies (AscTec) Falcon 8 Octocopter, equipped with an externally modified Sony Alpha NEX-7 camera (Table 1). The system weighs $2.3 \mathrm{~kg}$ (incl. camera), has a flat shape and can be transported to remote locations fully assembled in a special backpack, a prerequisite for most alpine applications.

Using exchangeable custom notch filters for the NEX-7 camera allowed to realize two consecutive flights within the ADS acquisition time slot, one flight with redefined RGB and the other flight with a NIR filter. The flight with NIR filter allows a limited comparison between the NIR band capabilities of the drone camera and the narrow NIR band of ADS100 data. (Bühler et al. 2016).

\begin{tabular}{|l|l|l|l|}
\hline Filter & Images & Height & GSD \\
\hline 830 & 85 & $153 \mathrm{~m}$ & $2.95 \mathrm{~cm}$ \\
\hline RGB & 85 & $157 \mathrm{~m}$ & $3.01 \mathrm{~cm}$ \\
\hline
\end{tabular}

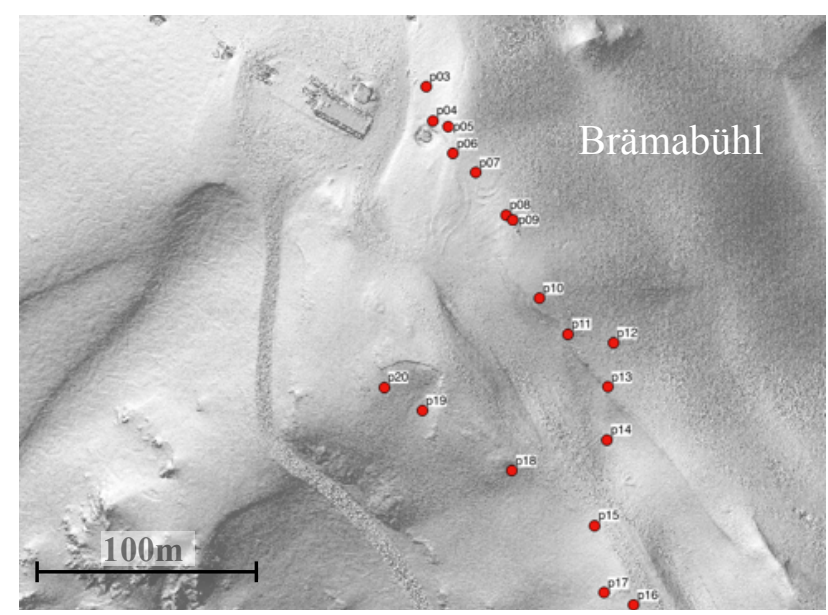

Figure 2. Shaded UAV DSM "Brämabühl" with reference points

\subsection{Reference Data}

Simultaneously with the ADS100 and UAV data acquisition, 20 different reference heights have been measured within the test site Brämabüel (Figure 2, Points 01-20). The spatial distribution of the point measurements were defined through the UAV flight plan, partially steep terrain and the actual snow coverage. Therefore points are not so evenly distributed as regularly as should be expected. A Trimble GeoXH differential GNSS with an accuracy of $10 \mathrm{~cm}$ was used in combination with GNSS correction data from the Swiss Positioning Service (swipos). During the measurement of 20 ground control points a PDOP of $2.1+/-0.8$ could be achieved.

\begin{tabular}{|l|l|r|l|r|}
\hline & $\begin{array}{l}\text { ADS80 } \\
\text { SH52 }\end{array}$ & width & $\begin{array}{l}\text { ADS100 } \\
\text { SH100 }\end{array}$ & width \\
\hline CCD & 12000 & & 20000 & \\
\hline pixelsize & $6.5 \mu$ & & $5 \mu$ & \\
\hline NIR & $833-887 \mathrm{~nm}$ & 54 & $808-882 \mathrm{~nm}$ & 74 \\
\hline Red & $608-662 \mathrm{~nm}$ & 54 & $619-651 \mathrm{~nm}$ & 32 \\
\hline Green & $533-587 \mathrm{~nm}$ & 54 & $525-585 \mathrm{~nm}$ & 32 \\
\hline Blue & $428-492 \mathrm{~nm}$ & 54 & $435-495 \mathrm{~nm}$ & 60 \\
\hline Resolution A/D & $12 \mathrm{bits}$ & & $14 \mathrm{bits}$ & \\
\hline backward & $16^{\circ}$ & & $18^{\circ}$ & \\
\hline forward & $27^{\circ}$ & & $25^{\circ}$ & \\
\hline GSD & $25 \mathrm{~cm}$ & & $15 \mathrm{~cm}$ & \\
\hline
\end{tabular}

Table 1. UAV acquisition configuration 
3.

\section{SENSORS AND METHODS}

\subsection{Camera Sensors}

The spectral and spatial resolution capabilities of an ADS100 sensor system compared to commercial DSLR cameras are very different, leading to different ranges of applications of these sensor systems. Therefore the comparison of achieved accuracies in elevation derivation between low-cost systems with small coverage and expensive systems with large coverage is important for cost-effective applications of aerial systems.

For the chosen test site NIR bands at nadir $\left(0^{\circ}\right)$ and backward $\left(18^{\circ}\right)$ have been selected, to achieve higher contrast and lower reflection on snow-covered areas (Bühler et al., 2015b).

The Sony Alpha NEX-7 system camera features a 24 MP APS$\mathrm{C}$ CMOS sensor and is equipped with a small and lightweight Sony NEX $20 \mathrm{~mm} \mathrm{~F} / 2.8$ optical lens $(81 \mathrm{~g})$. By removing the built-in low-pass filter, the camera sensor can be redefined using different notch filters to simulate visible $(\lambda<750 \mathrm{~nm}$ ("reconfigured" RGB)) and NIR band capabilities $(\lambda>550 \mathrm{~nm}$, $\lambda>770 \mathrm{~nm}$ and $\lambda>830 \mathrm{~nm}$ ). Specially the NIR sensitivity has advantages for different snow conditions (Bühler 2015b), as well as in diffuse light conditions. The camera is connected to the Falcon 8 by a gimbal with active stabilization and vibration damping and is powered by the UAV battery.

\subsection{Processing Workflow}

Image processing of ADS100 data requires specific software workflows, mainly due to the line strip geometry of the sensor data. On the other hand UAV images can be used with a large variety of photogrammetric software packages, but the less stable data acquisition leads to varying accuracies for inner and exterior orientation. Several publications have already investigated what snow height accuracy can be achieved with different photogrammetric images and workflows. But often the influence of several factors is not reproducible:

1. illumination conditions and snow granularity

2. sensor characteristic (spatial resolution, spectral range)

3. image matching method and parameters

Therefore this study considers the different workflows as a black boxes and focuses on the comparison of the resulting dense point clouds with manually acquired reference heights.

The ADS100 dense point clouds were created with SOCET SET ATE and SOCET SET NGATE using different processing parameters. The following parameter details summarize the different results:

\subsubsection{SOCET SET ATE v5.6.0}

4 different parameter variations have been calculated (AT $1 \mathrm{mSingle}$, AT $1 \mathrm{mMulti}$, AT $50 \mathrm{cmSingle}$, AT50cmMulti). With "Multi" 3 pairs of image stripes are used for processing, with "Single" only 1 image strip pair. All variations use the national DHM25 from swisstopo as seed-DTM. The final output resolution of $1 \mathrm{~m}$ allows the matching for $6-7$ pixels, with $50 \mathrm{~cm}$ resolution around 3-4 pixels are involved.

\subsubsection{SOCET SET NGATE v5.6.0}

4 different parameter variations have been calculated (NG1 mSingle, NG1mMulti, NG50cmSingle, NG50cmMulti). Similar to ATE, two output resolutions $50 \mathrm{~cm}$ and $1 \mathrm{~m}$ have been selected. With "Multi" 3 pairs of image stripes are used for processing, with "Single" only 1 image strip pair. Independent of the chosen final resolution, NGATE matches until the last pyramid level.

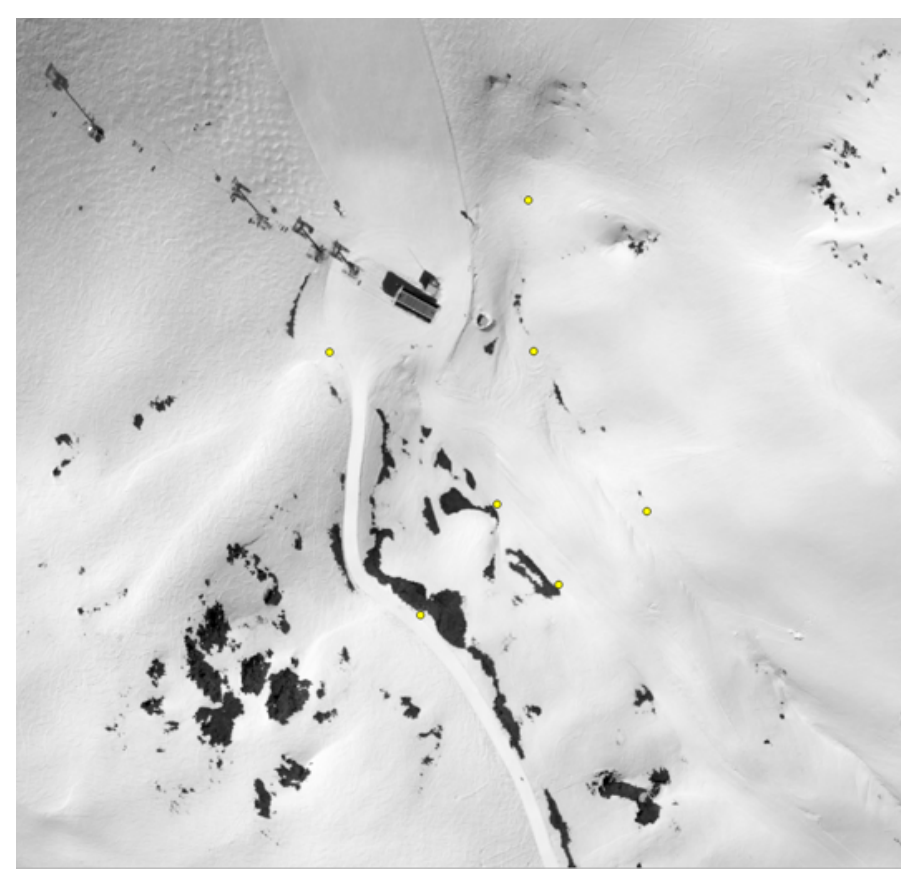

Figure 3. Orthoimage "Brämabühl" with 7 GCP points (yellow)

Both UAV flights (Table 1) have been processed using PhotoScan Professional v1.2.4 (PHS) and for the absolute orientation 7 ground control points (GCPs) have been used, which had to be positioned within the flatter part of the test site "Brämabühl" (Figure 3). The GCPs have been acquired with a Trimble GeoXH differential GNSS with an accuracy of $10 \mathrm{~cm}$.

The achieved overall accuracy errors of the absolute orientation with PHS are $8.5 \mathrm{~cm}$ for RGB images and $3.9 \mathrm{~cm}$ for $830 \mathrm{~nm}$ images.

\subsubsection{Photoscan Professional v1.2.4}

Building of the dense point cloud can be controlled with a point cloud depth filter "agg(ressive)", "mod(erate)" or "mil(d)". Therefore 3 different parameter variations for each camera filter have been calculated (PHAggRGB, PHModRGB, PHMilRGB and PHAgg830, PHMod830, PHMil830). 


\subsection{Results}

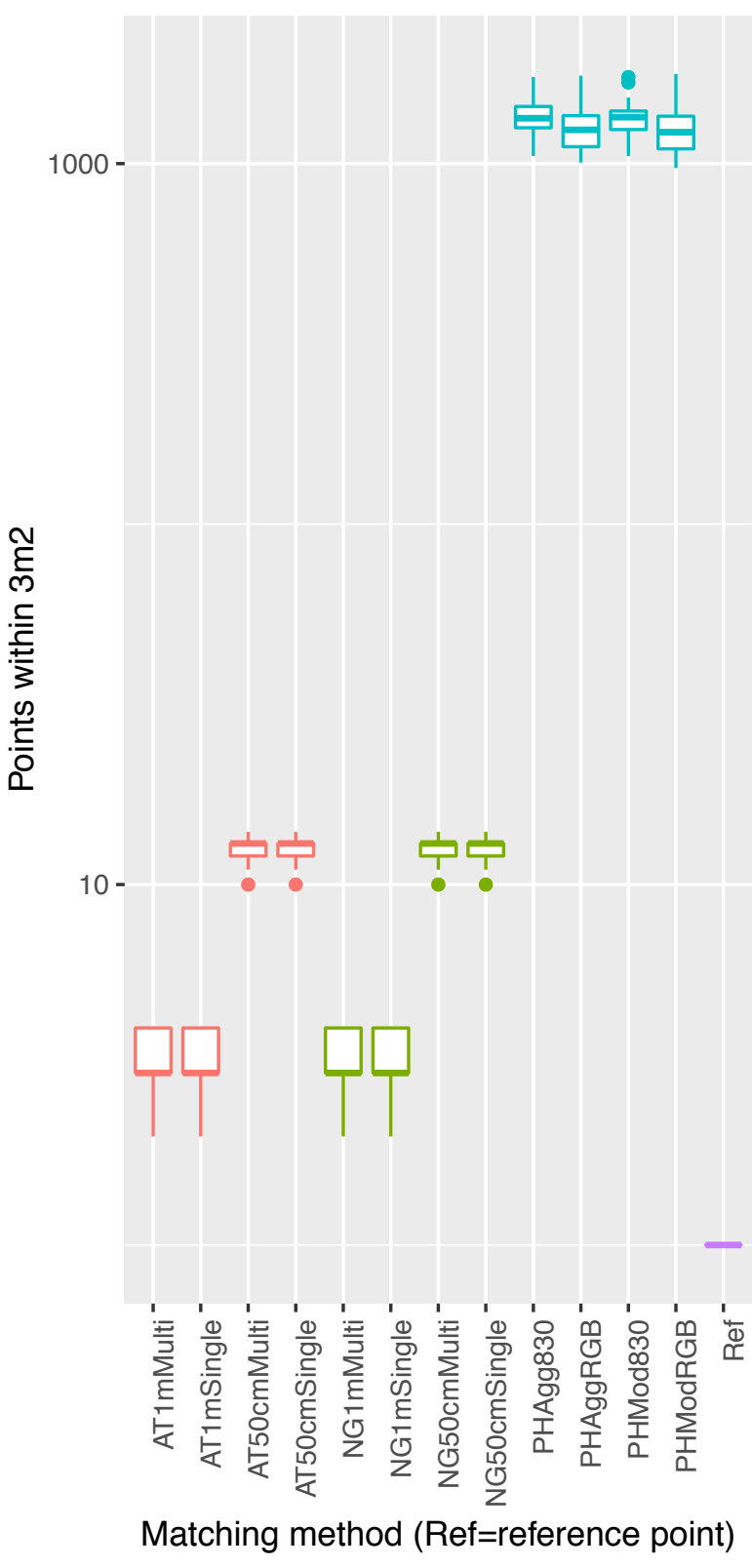

Figure 4. Extracted point clouds around reference points

To avoid resampling distortions, the dense point clouds for all 3 software packages have been used to compare snow height accuracy with the reference points. The rather wide search

radius of $1 \mathrm{~m}$ around each reference point is required (Figure 4), so that more than 1 point of every dense point cloud lies within the evaluation circle.

Figure 4 left reveals, that for $1 \mathrm{~m}$ resolution with SOCET SET ATE and NGATE results only 2-4 points are within each extracted $3 \mathrm{~m}^{2}$ point cloud.

Figure 4 right shows how much the extracted heights within the $3 \mathrm{~m}^{2}$ circle scatter, mainly due to local terrain undulations. $50 \%$ of all height values in the $3 \mathrm{~m}^{2}$ evaluation point cloud for all 20 reference points vary about $20 \mathrm{~cm}$ in height for SOCET SET ATE and NGATE. The high number of

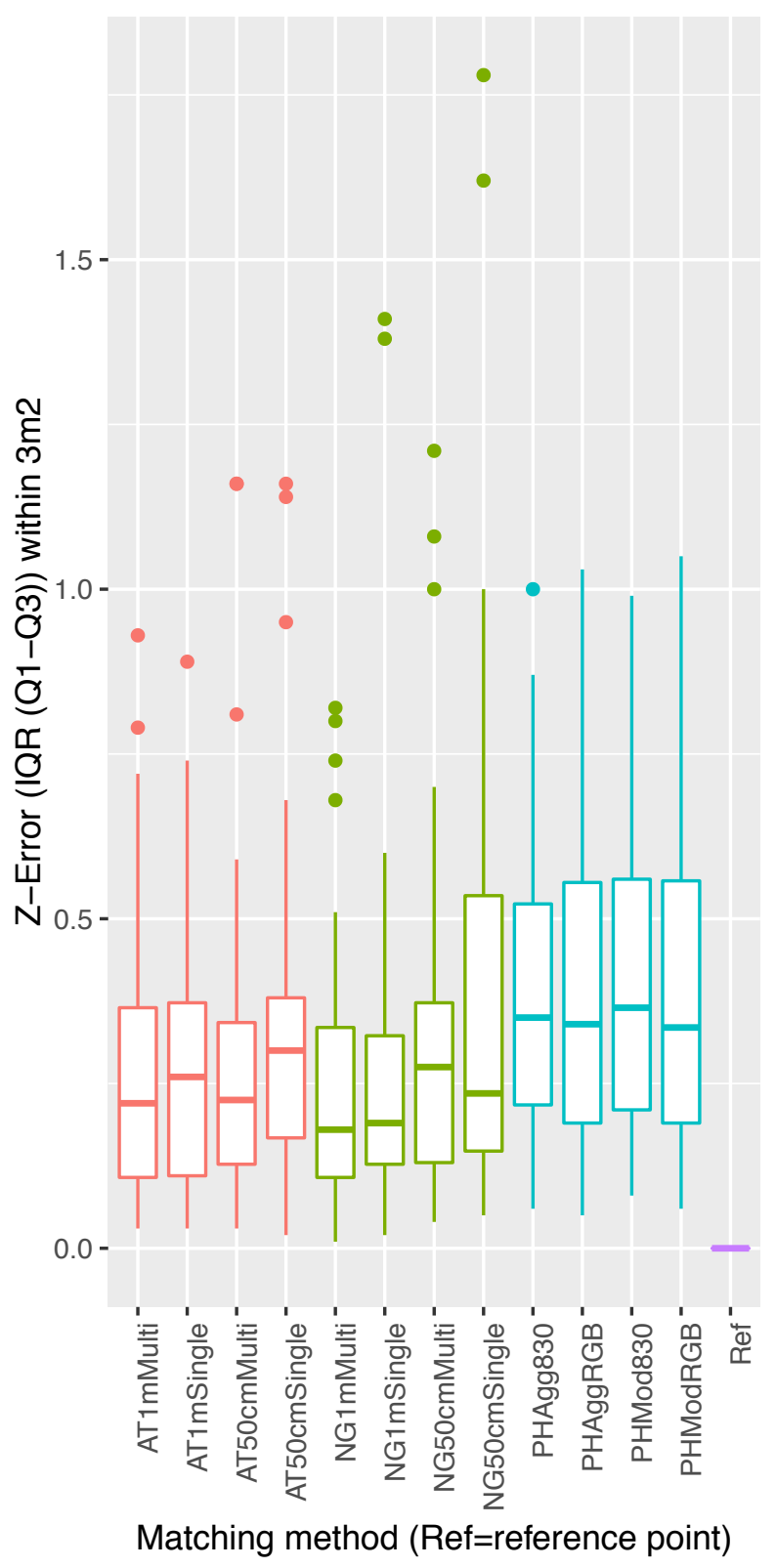

extracted points for PHS (> 1000) and several reference points with snow crests leads to an even slightly increased height IQR (blue boxplots in Figure 4 right (PHAgg830 ... PHModRGB)). Overall the height error of the reference points seem to be evenly distributed and each reference point is therefore equally representative. 


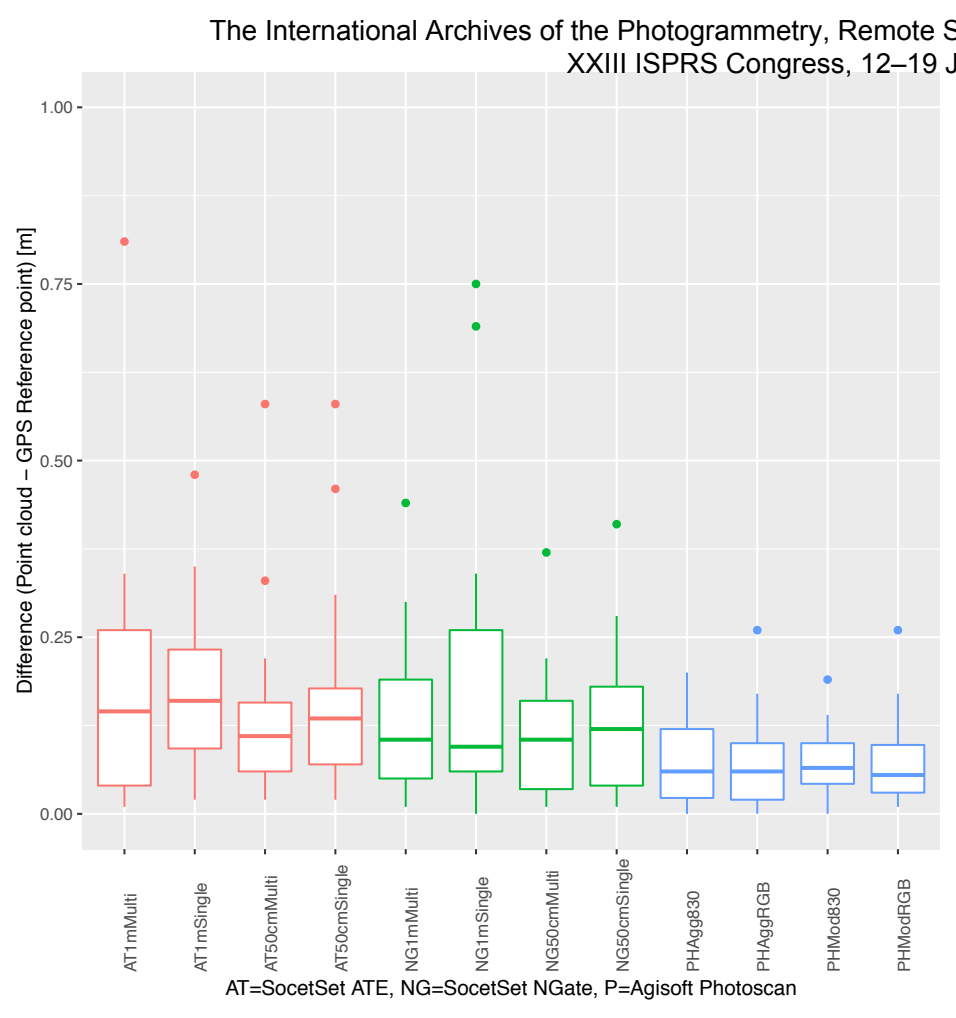

Figure 5. Difference (point cloud - reference point)

Each boxplot "Difference" (Figure 5) is calculated as

$$
\text { difference }=\sum_{i=1}^{n} \mid Z(\text { ref })-\operatorname{median}(Z(p c l)) \mid
$$

$\mathrm{n}=20, \mathrm{pcl}=$ point cloud, ref=reference point

The height evaluation of all reference points shows, that the better resolution of the PHS point clouds leads to a median height difference of 6-8cm (Fig. 5), which is close to the height error of the reference points themselves $(10 \mathrm{~cm})$. SOCET SET ATE and NGATE (all ADS100 point clouds depend on the same absolute orientation) reveal a larger median height difference of $10-17 \mathrm{~cm}$. In relation to the $15 \mathrm{~cm}$ GSD the ADS100 results are definitely improved compared with the results from 2012 (Bühler 2015a).

The different parameter variations in all processing workflows seem to have no significant influence. The difference results processed with SOCET SET or with PHS give no evidence, that computationally expensive options achieve better accuracies.

The results of SOCET SET ATE and NGATE are fairly similar, the median height difference for all NGATE variations are slightly lower than the ATE variations.

Aggressive filtering of all PHS dense point clouds (PHAgg830,PHAggRGB) has only little effect compared with moderate filtering (PHMod830, PHModRGB). Comparing RGB and $830 \mathrm{~nm}$ clouds does not show a significant height difference as well.

The single reference points in Figures 6 and 7 show a rather consistent deviation pattern. SOCET SET ATE and NGATE show for points 01-04 differences $10-22 \mathrm{~cm}$, for PHS the difference varies between $4-8 \mathrm{~cm}$, which corresponds to the ADS100 GSD of $15 \mathrm{~cm}$ and PHS GSD of $3 \mathrm{~cm}$.

Points 17-20 show an increased difference of $20-60 \mathrm{~cm}$ for SOCET SET ATE and NGATE, also for PHS with differences of $6-26 \mathrm{~cm}$. Steeper slopes and bumpier terrain at points $17-20$ could be main reason for the larger differences.

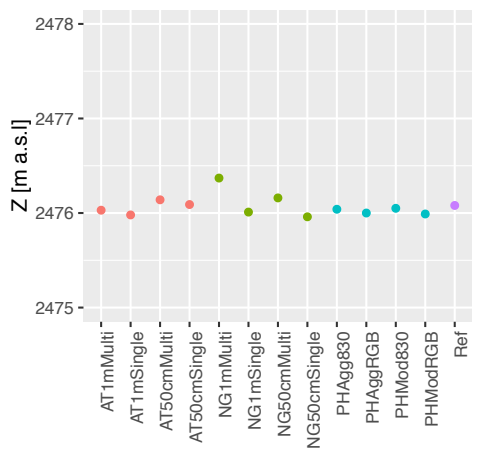

Reference point p02
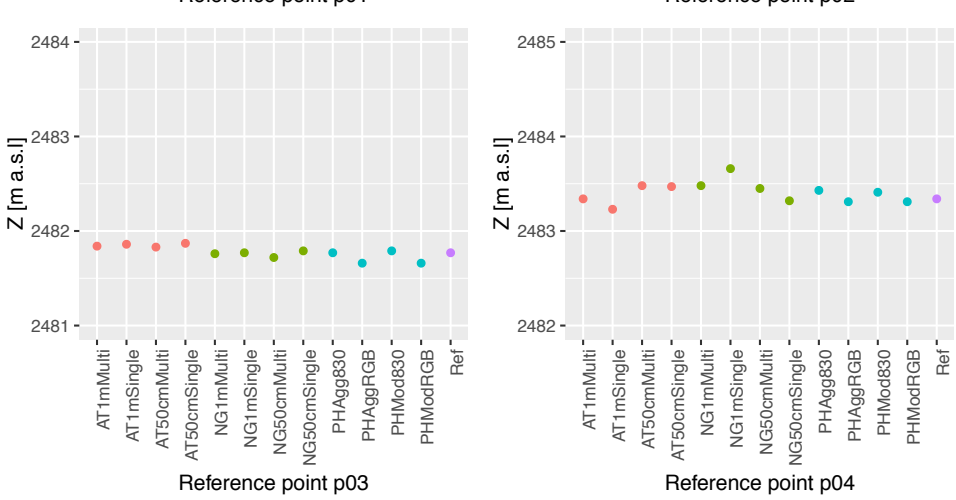

Reference point p04

Figure 6. Reference point 1-4 (reference height at the far right)
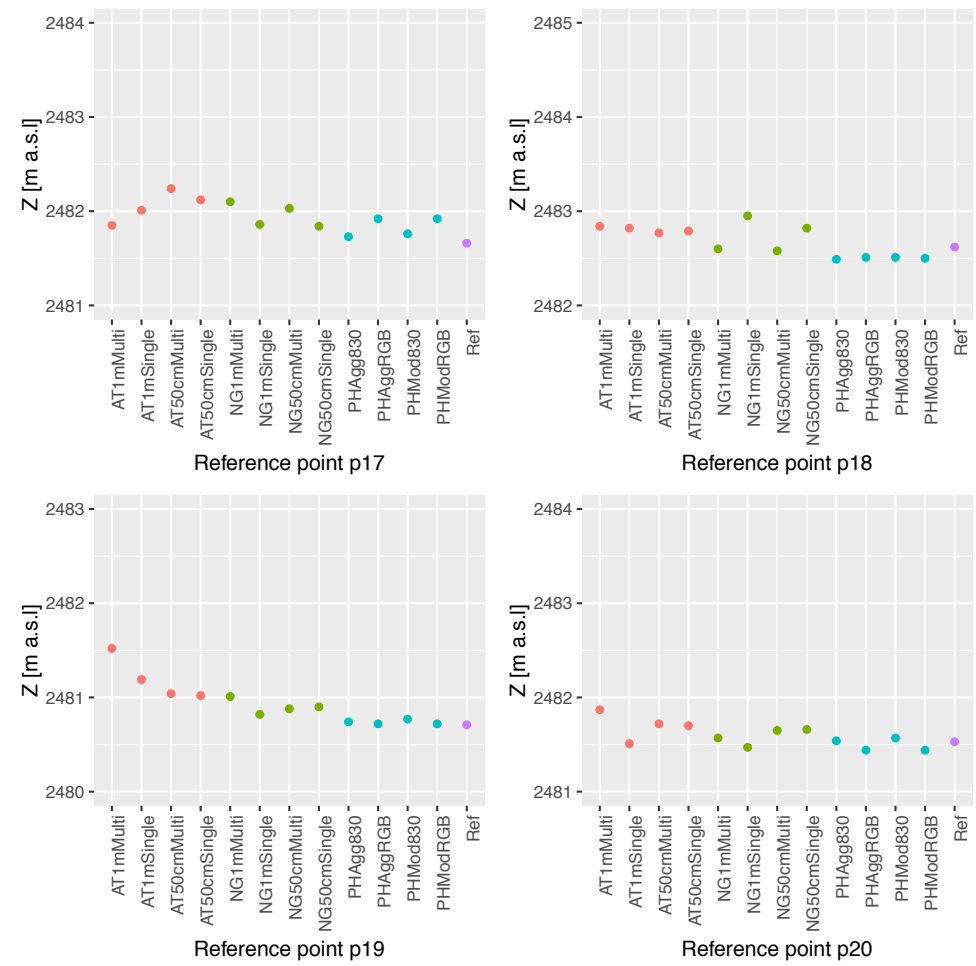

Figure 7. Reference point 17-20 (reference height at the far right) 


\section{Conclusions}

Snow height measurements with airborne sensors like the ADS100 and UAV-based DSLR cameras can achieve accuracies close to GSD * 2 in comparison with ground-based GNSS reference measurements. The improved spatial and spectral resolution for ADS100 is significant in comparison with ADS80 results (Bühler 2015a).

Using a custom notch filter on the camera sensor during UAV image acquisition does not yield better height accuracies, the better results with NIR band for ADS data (Bühler 2015a) cannot be reproduced with custom filtered APS-C sized images. Parameter variations for all workflows seem to have only random effects. For less perfect weather and illumination conditions this may change completely.

The height differences with SOCET SET ATE and NGATE are related mainly related to terrain slope and reduced illumination conditions, parameter variations for ATE and NGATE result often in contradictory effects (e.g. reference points $02,18,19$ ).

UAV based results with PHS are very robust and parameter variations have no significant effect. Therefore it can assumed, that image acquisition quality (sharpness and illumination), robust auto-calibration for the internal orientation and exact ground control points for the external orientation are more important than processing parameters in the following workflow.

The presented test was acquired during ideal weather conditions and fairly homogeneous snow conditions. Future research campaigns will show, what is the impact on snow height measurements with different atmospheric conditions as well as with dry or completely wet snow.

\section{ACKNOWLEDGEMENTS}

The authors would like to thank Leica Geosystems for the provision of the ADS100 data and the in-house staff of SLF for the support in the remote sensing investigations.

\section{REFERENCES}

Bühler, Y., Marty, M., Egli, L. et al: Snow depth mapping in high-alpine catchments using digital photogrammetry. Cryosphere, 9(1), pp. 229-243, 2015a.

Bühler, Y., Meier, L., and Ginzler, C.: Potential of operational, high spatial resolution near infrared remote sensing instruments for snow surface type mapping, Geoscience and Remote Sensing Letters, IEEE, 12, 821 - 825, $2015 \mathrm{~b}$.

Bühler, Y., Adams, M. S., Bösch, R., and Stoffel, A.: Mapping snow depth in alpine terrain with unmanned aerial systems (UAS): potential and limitations, The Cryosphere Discuss., 2016, 1-36, 2016.

DeVenecia, K., Walker, S., Zhang, B.: Photogrammetric Week. Institute of Photogrammetry "New Approaches to Generating and Processing High Resolution Elevation Data with Imagery", Stuttgart, Germany, 2007.

Lee, C. Y., Jones, S. D., Bellman, C. J., and Buxton, L.: DEM creation of a snow covered surface using digital aerial photography, The International Archives of the Photogram- metry, Remote Sensing and Spatial Information Sciences, 37, 2008.

Nolan, M., Larsen, C., and Sturm, M.: Mapping snow depth from manned aircraft on landscape scales at centimeter resolution using structure-from-motion photogrammetry, The Cryosphere, 9, 1445-1463, 2015.

Whitehead, K., Moorman, B. J., and Hugenholtz, C. H.: Brief Communication: Low-cost, on-demand aerial photogrammetry for glaciological measurement, The Cryosphere, 7, 1879-1884, 2013.

Zhang, B., Miller, S., Walker, S., DeVenecia K. Next Generation Automatic Terrain Extraction using Microsoft UltraCam imagery. Proceedings of the ASPS 2007 Annual Conference; Tempa, FL, USA. 7-11 May 2007. 